\title{
Le jongleur, mémoire de l'image. Figures, figurations et musicalité dans les manuscrits enluminés
}

M. CLOUzoT, Le jongleur, mémoire de l'image. Figures, figurations et musicalité dans les manuscrits enluminés, 1200-1330, Bern/Berlin/ Francfort-sur-le-Main/New York/Oxford/Vienne, éd. Peter Lang Verlag, 2011, 378 p.

\section{Martine Clouzot}

\section{OpenEdition}

Journals

Édition électronique

URL : https://journals.openedition.org/cem/12067

DOI : 10.4000/cem.12067

ISSN : 1954-3093

Éditeur

Centre d'études médiévales Saint-Germain d'Auxerre

Édition imprimée

Pagination : 451-455

ISSN : $1623-5770$

Référence électronique

Martine Clouzot, «Le jongleur, mémoire de l'image. Figures, figurations et musicalité dans les manuscrits enluminés ", Bulletin du centre d'études médiévales d'Auxerre | BUCEMA [En ligne], 15 | 2011 , mis en ligne le 25 août 2011, consulté le 22 septembre 2022. URL : http://journals.openedition.org/ cem/12067 ; DOI : https://doi.org/10.4000/cem.12067

Ce document a été généré automatiquement le 22 septembre 2022.

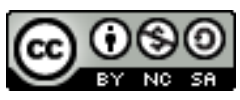

Creative Commons - Attribution - Pas d'Utilisation Commerciale - Partage dans les Mêmes Conditions 4.0 International - CC BY-NC-SA 4.0

https://creativecommons.org/licenses/by-nc-sa/4.0/ 


\section{Le jongleur, mémoire de l'image. Figures, figurations et musicalité dans les manuscrits enluminés}

M. CLOUZOT, Le jongleur, mémoire de l'image. Figures, figurations et musicalité dans les manuscrits enluminés, 1200-1330, Bern/Berlin/ Francfort-sur-le-Main/New York/Oxford/Vienne, éd. Peter Lang Verlag, 2011, 378 p.

\section{Martine Clouzot}

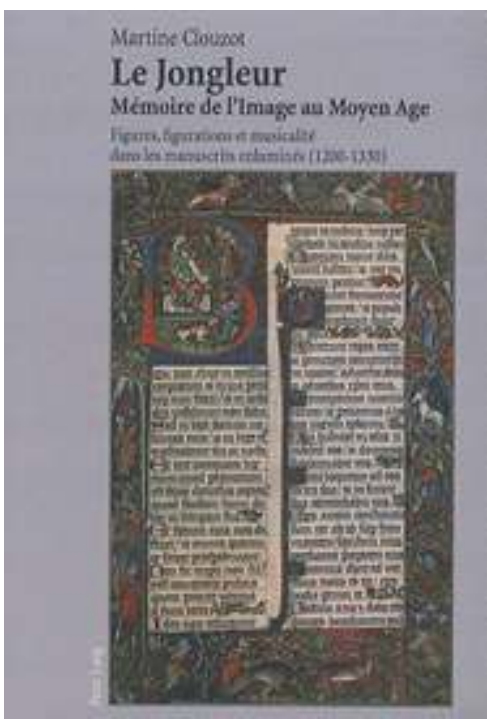

1 En couleur et en mouvement, les jongleurs jouent de la musique parmi les oiseaux, les animaux et les rinceaux fleuris dans les livres enluminés du Moyen Âge. Jamais loin du roi David, ils accrochent l'œil et éveillent l'esprit par leur caractère incongru et étonnant. Ce livre est consacré à leurs figurations dans les psautiers, les ouvrages de philosophie naturelle d'Aristote, destinés aux laïcs, dans les royaumes de France et d'Angleterre, entre 1200 et 1330 . Il étudie la transformation de la figure depuis 
l'Antiquité jusqu'à sa réinvention dans les milieux intellectuels au début du XIII ${ }^{e}$ siècle ${ }^{1}$. Il vise à montrer en quoi la figure du jongleur est une image de mémoire et une mémoire de l'image ${ }^{2}$, inscrite dans le corps de l'homme, sans cesse réactivée par la réminiscence et l'imagination, en vue d'une herméneutique spirituelle ${ }^{3}$.

Anthropologique ${ }^{4}$ et interdisciplinaire ${ }^{5}$, l'approche est croisée à l'histoire culturelle et aux sciences sociales. Elle n'étudie pas le jongleur en tant que « marginal » de la société médiévale. Les travaux menés dans cette perspective ne manquent pas. La démarche vise plutôt à analyser comment l'homme perçoit, conçoit et fabrique les figurations du jongleur dans des contextes donnés. C'est pourquoi cette étude est menée en interdisciplinarité avec l'histoire de l'art, de la musique, la philosophie, la littérature, ainsi que, dans une moindre mesure, la psychologie cognitive, la neurobiologie et les sciences du vivant.

3 Toute cette étude utilise et définit les deux termes " figure » et " figuration », car leurs sens, dans des registres différents, donnent les outils étymologiques utiles à de justes définitions de l'objet d'étude, le jongleur. Le terme «figure » est employé dans le sens littéral de forme extérieure d'un corps, ainsi que dans le sens rhétorique de forme de langage et de signe visible articulé au contenu. En cela, il rejoint le mot "figuration », qui, à la surface de la page enluminée, s'applique particulièrement aux formes visuelles du jongleur, à ses contours peints, tout en étant porteur de sens et de significations. Dans ce livre, le terme « figure » aura un sens générique, englobant la figure rhétorique et mnémonique, l'ornement graphique, le signe visuel et médium; "figuration » désignera plus spécifiquement l'image peinte, la forme visible aux contenus signifiants, et donc également le signe visuel médial.

\section{Les figurations}

Les figurations, tout d'abord. À un premier niveau de lecture, les rapports visibles entre le jongleur, la musique et le roi David sont sous-tendus par les conceptions physiques et biologiques sur le son et l'ouïe dans le cadre plus général des théories antiques et médiévales de la connaissance, en particulier chez Aristote dans la Métaphysique et le traité De l'âme, et saint Augustin dans le De Musica et les Confessions. À un niveau historique et généalogique, ces relations tiennent à trois causes essentielles: l'association du jongleur avec l'instrument de musique et le mouvement corporel dans les images; son inscription dans le psautier médiéval, livre poétique et musical de la Bible; ses liens formels, bibliques et mémoriels avec David, harpiste et danseur, roi et préfigure du Christ.

David, fils de Jessé, avant d'être roi, et de combattre le géant Goliath avec sa fronde, est, en effet, le jeune berger qui soulagea le roi Saül d'un mauvais esprit, de sa folie furieuse ${ }^{6}$. Puis, David est le roi qui ramena l'Arche d'Alliance de Dieu au son des instruments de « la maison d'Israël », qui dansa avec joie ${ }^{7}$. Lui-même, simplement ceint d'un " éphod de lin ", dansa, sauta, tourna sur lui-même devant l'Arche, sous le regard méprisant de Mical, la fille de Saül ${ }^{8}$. Par la danse, David rechercha l'humiliation volontaire devant Dieu ${ }^{9}$. Enfin, David, harpiste, est l'auteur des psaumes, poèmes prophétiques. Roi de l'Ancien Testament, David préfigure, par les psaumes, le roi du Nouveau Testament, le Christ. Le chant et les nombreux instruments de musique mentionnés en font des modèles exégétiques de spiritualité, de louange divine et de conversion. 
6 Les psautiers enluminés de la période 1200-1330 sont ornés de jongleurs, danseurs et acrobates, précisément sur les feuillets du premier psaume, le Beatus Vir, particulièrement étudié dans ce livre, mais aussi du Cantate Domino et de l'Exultate Deo, là où les mentions instrumentales sont nombreuses. Les relations visibles entre les figurations de l'instrumentiste, du roi harpiste et les psaumes interrogent sur la nature de leur musique : de la science musicale au chant psalmique, en passant par la physique du son et de l'ouïe, de quel medium et de quel mouvement herméneutique participentelles? Aussi, est-ce le terme "musicalité » qui a été retenu en sous-titre du livre. Inventé au début du $\mathrm{xx}^{\mathrm{e}}$ siècle, le terme étymologique signifie la qualité de ce qui est musical ou la ressemblance avec la musique ; le caractère musical s'inscrit ainsi dans une dynamique de la sensorialité, du corps et du langage. Aussi, ne s'agira-t-il pas de proposer une approche musicologique du jongleur, mais de considérer la musicalité, au sens corporel, sensible et linguistique, de ses figures et figurations.

\section{Les mots}

7 Les mots, ensuite, car les interprétations ont découlé de leurs mises en relations avec les images. Le terme français et générique "jongleur » a été retenu pour analyser les figures de joueurs d'instruments dans les marges des psautiers. Les raisons sont étymologiques, rhétoriques et historiques. La transformation du vocabulaire de l'histrio vers le joculator est observée conjointement dans les écrits et dans les images. Une étude lexicographique poussée montrerait, que, de façon très générale, comme Isidore de Séville les énumère au $\mathrm{VII}^{\mathrm{e}}$ siècle ${ }^{10}$, les histriones, les ioculatores antiques côtoient les mimi et les scurrae, les prestidigitateurs, les acteurs, les lanceurs d'épées, etc. ${ }^{11}$. La racine étymologique du joculator établit une première relation entre les mots et les images : jocus, $i$, nom masculin, signifie la plaisanterie, les farces et les jeux avec les mots. De là, les verbes et adjectifs jocari (plaisanter), jocosus (plaisant), joculari (dire des plaisanteries) et jocularis (risible, plaisant), joculosus - venant de jeu et jocista - qui joue avec les mots ${ }^{12}$, amplifient l'idée de jeu avec les mots. Proche de iocus, ludus concerne plutôt les jeux avec le corps et le délassement, notamment chez Thomas d'Aquin. Au pluriel, joci désigne les amusements et les jeux en général. Chez Quintillien, jocus définit le " contraire du sérieux ", associé à ce qui est ridicule, grotesque, obscène ${ }^{13}$.

8 Une continuité de sens de la racine jocus,entre les désignations du jongleur et sa figuration réinventée entre 1200 et 1330, guide cette étude. Entre l'Antiquité romaine et le XIII ${ }^{e}$ siècle, un glissement s'observe, en effet, entre les termes étymologiques et les figures peintes. D'un côté, l'emploi conjoint des mots histrio et joculator au début du XIII ${ }^{\mathrm{e}}$ siècle, est marqué par l'abandon progressif d'histrio à partir de la seconde moitié du siècle, en latin et en langues vernaculaires. D'un autre côté, les figurations du jongleur, plaisantes, animées et colorées, émergent dans les livres peints, comme si le jocus était recomposé et amplifié dans les images et par l'emploi renouvelé des termes, tels que ioculator, jogleor, gengleur, joglar, etc. dans les chansons de geste, les sermons et les traités.

9 L'activation de la mémoire et la réminiscence au moyen des mots, des paroles et des images étonnantes seraient la signification visible et principale des figurations du jongleur dans les livres. Car, parmi les typologies et les références, pourquoi les auteurs, clercs et opérateurs d'images, ont-ils choisi cette figure plutôt qu'une autre ? À partir de quelles traditions culturelles et de quels modèles de transmission ont-ils 
fabriqué et transformé une nouvelle figure du jongleur, en image mnémonique d'une historia christologique et herméneutique?

Trois chapitres, sous-tendus par les relations interactives entre le jongleur musicien et le roi David harpiste, visent à reconstituer les réseaux de transmission culturelle, qui, depuis l'Antiquité, ont contribué à forger la figure du jongleur entre 1200 et 1330 . Le premier chapitre retrace une histoire culturelle du jongleur depuis l'histrio grécoromain, jusqu'au jongleur $\mathrm{du} \mathrm{xIII}^{\mathrm{e}}$ siècle. Le second chapitre s'intéresse plus particulièrement aux figurations du jongleur dans les livres peints, d'une part les psautiers (1200-1330), d'autre part de philosophie naturelle d'Aristote: il confronte les figurations aux conceptions du son et des objets sonores entre 1270 et 1330. Enfin, le troisième chapitre analyse la figure du jongleur comme signe et médium, image de mémoire et mémoire d'une image, celle du Christ, inscrite dans l'âme et le corps de l'homme, depuis le temps biblique de la Création.

11 Ce livre a été un point de départ et d'arrivée. Concluant plusieurs années de recherches sur les musiciens dans les manuscrits peints et les archives, sa composition a ouvert des champs nouveaux d'explorations. Pour son auteure, il est désormais une étape vers d'autres études sur les figurations médiévales des objets sonores et leurs rapports avec le langage, la folie et la nature.

\section{NOTES}

1. J. LE GOFF, Les intellectuels au Moyen Âge, Paris, 1985 ( $1^{\text {re }}$ éd. 1964).

2. H. HAJDU, Das Mnemotechnische Schriftum des Mittelalters, Budapest, 1936 ; F. A. YATES, L'art de la

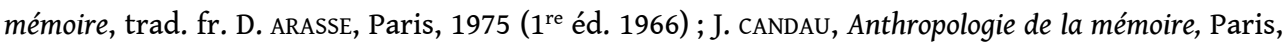
1996 ; M. CARRUTHERS, Machina Memorialis. Méditation, rhétorique et fabrication des images au Moyen

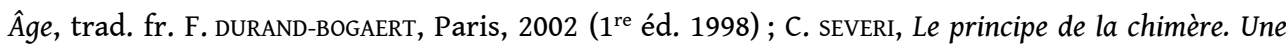
anthropologie de la mémoire, Paris, 2007 ( $1^{\text {re }}$ éd. 2004).

3. G. DAHAN, L'exégèse chrétienne de la Bible en Occident médiéval. XII ${ }^{e}-X I V^{e}$ siècle, Paris, 1999.

4. H. Belting, Pour une anthropologie des images, Paris, 2004 ( $1^{\text {re }}$ éd. 2001),p. 18: « Le discours anthropologique n'est pas assujetti à une discipline spécifique, mais a pour projet d'appréhender l'image sur un mode interdisciplinaire et ouvert »; J.-C. sCHмITT, " La culture de l'imago », Images médiévales, Annales H.S.S., janvier 1996, p. 3-36 et ID., Le corps des images. Essais sur la culture visuelle au Moyen Âge, Paris, 2002.

5. J. FRIED, DerSchleier der Erinnerung. Grundzüge einer historischen Memorik, Munich, 2004.

6. I Sam. 16, 23: "Quand donc l'esprit mauvais fondait sur Saül, David prenait la harpe et la pinçait. Saül se calmait, il était soulagé et le mauvais esprit le quittait. »

7. II Sam. 6, 5: «David et toute la maison d'Israël dansaient de toutes leurs forces devant le Seigneur et chantaient, accompagnés de harpes et de cithares, de tambourins, de sistres et de cymbales. »

8. II Sam. 6, 13-17: "Quand les porteurs de l'Arche du Seigneur eurent fait six pas, il sacrifia un taureau et un veau gras. David dansait de toutes ses forces devant le Seigneur ; il était ceint d'un éphod de lin. David et tous les Israélites poussaient des cris de joie et sonnaient de la trompe, tout en faisant monter l'Arche du Seigneur. Quand elle pénétra dans la Cité de David, Mical fille de 
Saül, qui regardait par la fenêtre, aperçut le roi David sautant et tournant sur lui-même devant le Seigneur, et elle conçut pour lui du mépris. »

9. II Sam. 6, 21-23: "C'est devant le Seigneur que j'ai dansé ! répliqua David. Par la vie du Seigneur qui m'a choisi de préférence à ton père et à toute ta famille pour me faire le chef de son peuple Israël, je danserai encore devant le Seigneur. Et je m'abaisserai davantage encore, dussé-je m'avilir à tes yeux, tandis que je vais me faire apprécier par les servantes dont tu parles. »

10. ISIDORE DE SÉVILLE, Étymologies, Livre XVII, De bello et ludis, $43:$ « De Scena. Scena autem erat locus infra theatrum in modum domus instructa cum pulpito, qui pulpitus orchestra vocabatur; ubi cantabant comici, tragici, atque saltabant histriones et mimi. Dicta autem scena Graeca appellatione, eo quod in speciem domus erat instructa. Unde et apud Hebraeos tabernaculorum dedicatio a similitudine domiciliorum $\sigma \kappa \varepsilon v o \pi \eta ́ \gamma l \alpha$ appellabantur.» Isidoreregroupe ainsi trois grands types d'intervenants dans les jeux de scène : les acteurs (comici et les tragici), qui chantent; les acteurs (saltatores, les histriones et les mimi), qui dansent et imitent; et les musiciens (les thymelici) jouant des instruments.

11. J. D. A. OGILVY, « Mimi, Scurrae, Histriones : Entertainers of the Early Middle Ages ", Speculum, 38 (1963), p. 603-619.

12. Le sens de jocus évoluera lui aussi, pour servir, au Moyen Âge, à désigner le jeu hippique, la joute, tandis que joculosus évoluera, pour se recentrer autour du sens de railleur (cf. Niermeyer et Du Cange).

13. J. LECLERCQ, «Ioculator et saltator. Saint Bernard et l'image du jongleur dans les manuscrits », in Translatio studii. Manuscripts and Library Studies honoring O.L. Kapsner, osb, Collegeville, 1973, p. 124-148, ici p. 125.

\section{INDEX}

Mots-clés : figuration, figure, image, jongleur, manuscrit enluminé, musicalité 